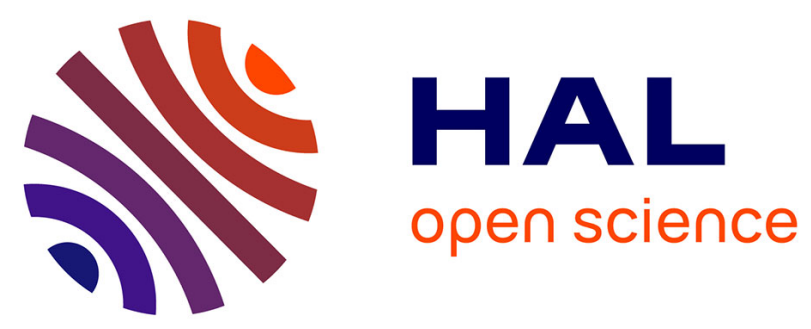

\title{
The Concepts of Decision Making: An Analysis of Classical Approaches and Avenues for the Field of Enterprise Modeling
}

Alexander Bock

\section{- To cite this version:}

Alexander Bock. The Concepts of Decision Making: An Analysis of Classical Approaches and Avenues for the Field of Enterprise Modeling. 8th Practice of Enterprise Modelling (P0EM), Nov 2015, Valencia, Spain. pp.306-321, 10.1007/978-3-319-25897-3_20 . hal-01442258

\author{
HAL Id: hal-01442258 \\ https://hal.inria.fr/hal-01442258
}

Submitted on 20 Jan 2017

HAL is a multi-disciplinary open access archive for the deposit and dissemination of scientific research documents, whether they are published or not. The documents may come from teaching and research institutions in France or abroad, or from public or private research centers.
L'archive ouverte pluridisciplinaire HAL, est destinée au dépôt et à la diffusion de documents scientifiques de niveau recherche, publiés ou non, émanant des établissements d'enseignement et de recherche français ou étrangers, des laboratoires publics ou privés.

\section{(c)(1)}

Distributed under a Creative Commons Attribution| 4.0 International License 


\title{
The Concepts of Decision Making: An Analysis of Classical Approaches and Avenues for the Field of Enterprise Modeling
}

\author{
Alexander Bock \\ Information Systems and Enterprise Modeling Research Group \\ University of Duisburg-Essen, Essen, Germany \\ alexander.bock@uni-due.de,
}

\begin{abstract}
The field of enterprise modeling seeks to help make organizational decisions that take place under conditions of complexity, ambiguity, and conflicting views. But available concepts to describe decision situations are still in their infancy. A variety of existing model-based approaches to aid decision making have been developed in adjacent research fields. The paper investigates a selection of these approaches and reconstructs central concepts in the form of meta models. Implications and ways of integrating decision concepts with existing enterprise modeling methods are considered, and possible avenues for the field of enterprise modeling are discussed.
\end{abstract}

Key words: Organizational decision making, enterprise modeling, decision model, decision analysis, problem construction.

\section{Introduction}

The conditions within which organizational decisions must be made are complex, ambiguous, and conflict-laden [1]. Decisions in organizations happen in view of conflicting goals [2], contradictory problem definitions [3], and increasingly volatile markets and environments. In consequence, it is long apparent that decision makers in organizations can profit from support (e.g., [2] [4]).

The field of enterprise modeling (EM) seeks to offer conceptual means to describe, integrate, and analyze multidisciplinary views of an organization [5, pp. 942-943] [6, pp. 18-20]. It is conventionally argued that enterprise modeling can aid decision making. Help is supposed to be offered by virtue of explicating links between different areas of an enterprise, stimulating multidisciplinary discussions, and enabling various domain-specific analyses (see, e.g., [5, pp. 943944] [6, pp. 9-20]). But although this sort of decision support is implicit in the notion of enterprise modeling, common methods such as For Enterprise Modeling (4EM) [6], ArchiMate [7], or Multi-Perspective Enterprise Modeling (MEMO) [5] offer few, if any, specific concepts to describe decision situations.

The lack of decision-related concepts in enterprise modeling has already been recognized, and some suggestions have been made as to the extension of existing methods with decision concepts (see, e.g., [8] [9] [10]). Incorporating decision 
models in enterprise models may be useful in several ways. For example, integrated models could help document and evaluate the determinants considered in certain types of recurring decision processes (see [8]). This could contribute to a continuous (re-)assessment of decision practices in the organization - an effort whose practical relevance has come to recognition (see [4] [11]). It would also be possible to model and analyze particular decision situations. This would address aims similar to those of known kinds of decision models (see, e.g., [12, pp. 105112]) but with an enhanced account of the organizational context. Furthermore, the models could be used to document particular past decisions (see [9]). But while previous research has indicated these and other benefits, most existing contributions are still in an early stage of development.

Meanwhile, using models to support decision making has a long tradition. Different research fields have long been developing and putting to use modelbased approaches to analyze decision situations. That includes research fields such as decision theory and analysis [2] [13] [14] [15], systems thinking research [16] [17], management and consulting studies [3] [12], and more remote fields such as political studies [18]. Example approaches include decision trees, influence diagrams, cognitive mapping, and Soft Systems Methodology (SSM) [16] [17]. In view of their different backgrounds, it can be assumed that these existing approaches, when taken together, offer a wide variety of different concepts to make sense of decision situations.

In order to advance research on incorporating decision concepts in enterprise modeling, this paper seeks to contribute to the development of a broader perspective on possible concepts to describe decision situations. The paper makes two contributions. One is to obtain a set of decision-related concepts which have been discussed extensively in a rich body of existing research. This is done by reviewing a selection of classical decision modeling approaches and reconstructing their concepts in the form of meta models. Second, implications and prospects for the field of enterprise modeling are formulated and discussed to indicate research avenues for the development of decision modeling extensions.

The paper proceeds as follows. In section 2, selected theoretical lines of decision research are summarized. The current state of decision concepts in the field of EM is assessed in section 3. Classical decision modeling approaches are analyzed in section 4. Implications and possible avenues are discussed in section 5. The paper closes with a call for further research in section 6 .

\section{Theoretical Views on Decisions}

Human decision making and problem solving processes are investigated in many disciplines. To prepare the discussion in section 4, selected insights of descriptive decision research are briefly visited. What is a decision? Ordinary definitions in many research fields state that it is a choice among a set of alternatives [1, pp. 1011] [19, p. 11]. An individual who must make a decision is thought to have to choose between a set of alternative courses of action. A good or "optimal" choice, according to this view, is seen as one that maximizes the presumed attainment 
of pursued goals [1, p. 11]. This view is shared in wide parts of various research fields, including psychology (see, e.g., [20, pp. 656-657] [21, pp. 568-572]) and decision analysis (see, e.g., [2, pp. 806-807]). The choice view directs attention at some aspects of decision making and neglects others. What it neglects is that neither the full context of a decision situation, let alone all possible courses of action [22, p. 81], may be readily apparent to an individual as soon as a need to make a decision is perceived. Rather, these aspects have to be explored and constructed gradually [20, p. 658]. In consequence, decisions have been recognized to emerge from perhaps lengthy processes [23, pp. 39-40] [24, p. 246].

How does a decision process unfold? Many theories exist. It has been argued that a decision process is initiated as soon as an individual perceives that a situation warrants action but it is not obvious what kind of action may be adequate (cf. [19, p. 127] [23, p. 102]). In this connection, it is often distinguished between situations that are more routinized or rule-driven and situations that require conscious deliberation and reflection (e.g., coined 'programmed' and 'non-programmed' decisions by [23, p. 46]). Situations of the latter kind are commonly referred to as 'problems' (see, e.g., [25] [17] [26]). However, it has been contended that a situation may be seen as a 'problem' only inasmuch as an individual construes it as one $[3$, p. 8$][19$, p. 127]. In this sense, problems have become understood as human constructions rather than as objective entities [27, pp. 326-330] [3, p. 8] [26, p. 35]. The argument may also be relevant when considering common distinctions between 'well-structured' and 'unstructured' problems, where the determinants of the former are thought to be clear in advance, while those of the latter require investigation (cf. [27, pp. 321-330]; see also [25, p. 4-9]). As has been pointed out, even the structure of 'structured' problems is not a property of an objective situation, but the result of a human interpretation [27, pp. 321-332] [26, p. 35]. Once a decision process is initiated, a plethora of modes of behavior and cognitive processes have been hypothesized to be functional, including information search, solution generation, and various evaluation and choice heuristics and strategies (see, e.g., [21, pp. 581-601] [20, pp. 658-674]). In case an acceptable course of action is identified, a decision process may result in a decision - not necessarily in the sense of choice, but as a commitment to this course of action [4, p. 24] [24, p. 246]. Finally, matters become more complicated when considering collective decision processes. Individuals in organizations are working in a social context. In consequence, it has also been argued that problems, in the process of being analyzed and discussed, are socially constructed (see, e.g., [3] [25]). But aside from a collaboration-centric dimension, the social context is also recognized to add modes of behavior such as power exertion, manipulation, and bargaining (e.g., [1] [3]).

\section{Decisions in EM: Current State and Related Work}

Enterprise models are intended to describe both sociological-organizational and informational-technological facets of an enterprise in a manner which points out the interleaving between them [5, pp. 942-943] [6, p. 29]. It would therefore 
seem plausible to expect that EM approaches offer concepts to describe decision processes and problem situations in the organizational context. In this section, it is reviewed whether this is so. First, existing EM methods are reviewed. Second, attention is directed at specific decision modeling extensions.

General enterprise modeling methods. A variety of EM methods exists (for recent overviews, see [6, pp. 233-272] [28]). Three common example methods that specifically aim to cover different areas of an enterprise are For Enterprise Modeling (4EM) [6], ArchiMate [7], and Multi-Perspective Enterprise Modeling (MEMO) [5]. These are briefly reviewed to indicate conventional concepts in the field. $4 E M$ does not provide a specific decision concept. But concepts are offered to create 'goals models', which most importantly encompass the concept of a 'goal' [6, p. 88]. In addition, 'goals models' may include the related concepts 'problem', 'cause', 'constraint', and 'opportunity' [6, p. 88]. In the context of business process models, an 'OR' split concept can be found which can be construed as a decision concept in the sense of a choice [6, p. 121]. ArchiMate [7] also does not offer a 'decision' concept. Decision-related concepts can be found in the motivation extension [7, pp. 141-152]. These are essentially normative in nature, including the concept of a 'goal' plus the related concepts 'driver' and 'requirement' [7, pp. 143-148]. There are also the concepts 'constraint' and 'principle' [7, pp. 149-151]. MEMO is an enterprise modeling method that comprises a set of integrated modeling languages to describe different views on an enterprise [5, p. 946]. The goal concepts 'EngagementGoal' and 'SymbolicGoal' can be found in a comprehensive goal modeling language [29, pp. 10-12]. Further, in the context of a business process modeling language, the concept 'branching' is defined to describe branching points in processes [30, p. 56]. Its attribute 'decision' can be used to indicate whether that path choice is rule-driven or requires human expertise [30, p. 54]. Decision-related aspects are also implicit in various attributes such as 'justification' (see, e.g., [29, p. 11]). Recently, an extension to describe decision processes has been suggested [8], which is mentioned below.

In sum, common EM methods mainly offer decision concepts in four respects (although the level of detail and elaborateness varies significantly). (1) They offer goal and other teleological concepts; (2) they offer exclusive path choice concepts for business processes; (3) they offer coarse concepts related to problems; and (4) they can document various kinds of decisions implicitly by means of justificatory or explanatory attributes. What is missing, however, are dedicated concepts to describe decision situations or decision processes in detail.

Specific decision modeling approaches. In view of the situation indicated above, some extensions to model decisions in the context of enterprise models have been suggested. An approach to document the rationales for design decisions in enterprise architecture (EA) management is developed by [9]. The approach mainly takes an ex-post view of decisions. Its main concepts include 'EA Decision', 'EA Issue', 'Decision-Making Strategy', 'Strategy Rationale', 'Criterion', and various relationships including 'Decomposition' and 'Alternative' [9, pp. 135-137]. Instances of these concepts can be linked to elements in EA models to explain how they have been arrived at [9, pp. 135-137]. A domain-specific 
modeling language as an extension to EM methods (in particular, MEMO) has been proposed in [8]. It aims to permit modeling organizational decision process types plus their context from various perspectives [8, pp. 183-186]. Its main concepts to describe problem situations include 'DecisionPremise', 'ValueDecisionPremise', 'AbstracGoal' (resued from [29]), 'FactualDecisionPremise', 'ActionVariable', 'SituationalAspect', 'EnvironmentalFactor', and relationships such as 'RelevanceRelation' and 'PresumedInfluenceRelation' [8, pp. 187-189]. The approach intends to support continuous reflections about the premises as well as the organizational-regulatory and information-technological conditions of decision processes [8, pp. 183-186]. As an industry-driven initiative, the Decision Model and Notation (DMN) has been suggested by the Object Management Group (OMG) [10]. It permits to describe the logic and rationale of decisions and link them to business processes and organizational units [10, pp. 23-53]. Its underlying definition of a decision as an "act of determining an output value [...], from a number of input values, using logic" [10, p. 23] makes it clear that the focus of DMN is placed on more routine operational decisions (cf. [7, p. 27]). The provided concepts include 'Decision', 'InformationRequirement', 'KnowledgeSource', 'InputData', and various relationships [10, pp. 37-53]. Comprehensive mechanisms are provided to specify the rules for choosing among given input values, including decision tables and an expression language [7, pp. 70-141].

Wrapping up the discussion, it can be summarized that work is in progress to augment enterprise modeling approaches with decision concepts. Even in this early stage, the existing contributions indicate a considerable range of possible uses for such concepts. However, as the development of each approach sets out from an individual starting premise, the respective publications do not discuss markedly different concepts to describe decision situations. In particular, while there seems to be a minimal consensus on concepts to describe more clear decision situations (e.g., alternatives and criteria), only a few broader concepts (e.g., 'SituationalAspect'; [8]) are available to describe decision situations that might be interpreted as less structured. In the following section, this paper seeks to contribute to advancing research in this direction by intending to unveil longstanding, yet varied decision concepts developed in different research fields.

\section{Classical Decision Modeling Approaches}

Different model-based approaches to facilitate decision making and problem solving have been proposed in the literature and advanced in practice. A selection of such tools are reviewed and discussed below. The approaches have been selected in view of two criteria. First, focus is placed on tools which have found considerable use and reception practice and academia. Second, as a contrast to the first criterion, the tools were selected such as to be deliberately distinct and varied, in terms of the scientific background, the underlying assumptions, and the specific concepts used. In particular, the tools have been taken from traditional decision analysis as well as from skeptical movements in other fields (for comparative discussions, see [12, pp. 105-111] [25]). The discussion centers around concepts 
to describe decision situations or problems as such. Other relevant aspects, such as organizational regulations or psychological phenomena, are not considered. The central concepts of the approaches are reconstructed in the form of meta models (at level $M_{2}$ ) using the MEMO meta modeling language (MML) [31]. The main purpose of the meta models is to point out central concepts; the meta models do not suffice to specify fully correct models of the respective approaches (additional constraints are omitted as well). Furthermore, as classical modeling approaches do not follow the exact same abstraction patterns as are common in the field of EM, the abstraction levels are not determined unambiguously.

\subsection{Concepts in Classical Decision Analysis}

There is an abundance of work on decision models in the fields of decision theory and analysis (for overviews, see, e.g., [2] [13] [14] [15]). But although advanced mathematical and probability theory is applied, it is commonly asserted that decision situations are essentially apprehended through the lens of a basic framework (see, e.g., [14, pp. xxii-xxiii] [15, pp. 13-22]). The elements of this framework resemble the choice view mentioned in section 2 and can be identified as follows. (1) There is a set of alternatives among which a decision maker must choose (see, e.g., [2, p. 807] [13, pp. 1-2] [15, pp. 15-18]). It is often distinguished between discrete (e.g., different products to be purchased) and continuous alternatives (e.g., the amount of financial budgets) [13, p. 1]. In case different aspects can be varied the full alternative space is the set of all combinations of decision variable values. (2) Perhaps, there are environmental factors which can take different states in the future [14, pp. xxii-xxiii]. This is sometimes referred to as uncertainty or risk in general (cf. [2, p. 807]). (3) There is a set of outcomes (or consequences), each of which is linked to a combination of an alternative and an environmental state (see, e.g., [2, p. 807]). (4) To evaluate outcomes, it is assumed that there is at least one and perhaps several goals (or objectives or criteria) (see, e.g., [2, p. 807] [13, p. 2]). To be able to quantitatively evaluate outcomes with respect to goals, goal fulfillment values have to be defined. Sometimes the outcome itself is already taken to represent the extent of goal fulfillment (e.g., in the case of profit figures which are the better the greater they are). To represent formal models, specific representational forms have been developed. A selection of common examples is discussed below.

Decision matrices (or tables) are one of the most common and elementary representational form used in the field of multi-criteria decision analysis (see, e.g., [13, pp. 2-3]). Different variants exist. One customary variant assigns outcome values to particular combinations of an alternative, environmental state and/or goal (cf. [15, p. 27]). An example is shown in figure 1. It is apparent that a decision matrix displays concrete values for the concepts 'goal', 'environmental state', 'alternative', and 'outcome'. Note that the alternative values must be fully disjunctive. Figure 2 shows a reconstructed meta model for decision matrices.

Decision trees are another ordinary representational form used in decision analysis (see, e.g., [14, pp. 10-17] [15, pp. 242-243]). The basic purpose of decision trees, again, is to assign outcome values to all combinations of alternative 


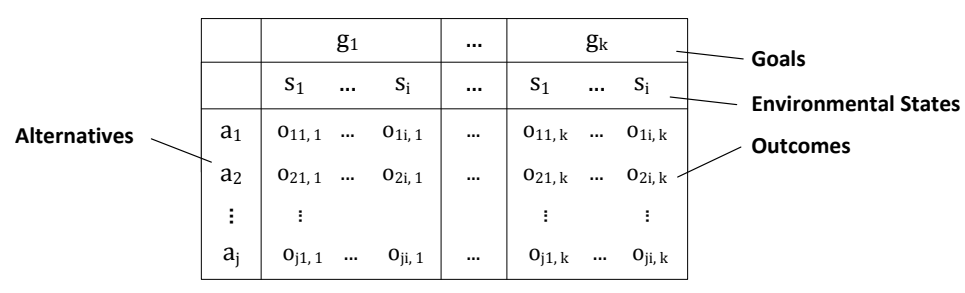

Fig. 1. Example decision matrix

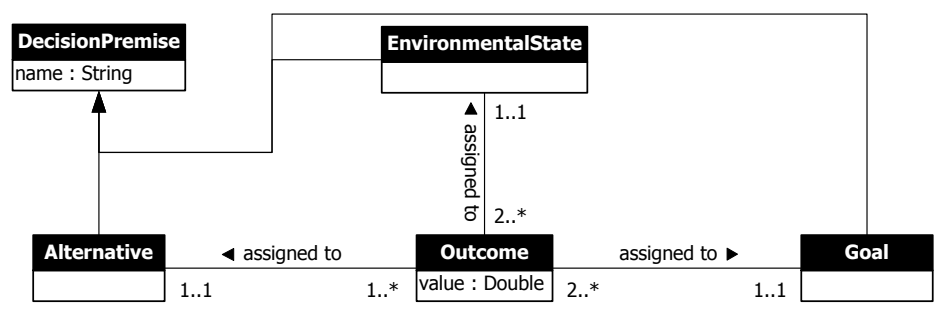

Fig. 2. Reconstructed meta model of decision matrices

and environmental states. Contrary to decision matrices, however, it is possible to divide one fully disjunctive alternative space into alternatives for several sequential decisions [15, p. 242]. Decision trees are tree-like graphs that consist of three kind of nodes: Decision nodes, chance nodes, and outcome nodes. Figure 4 shows a reconstructed meta model for decision trees.

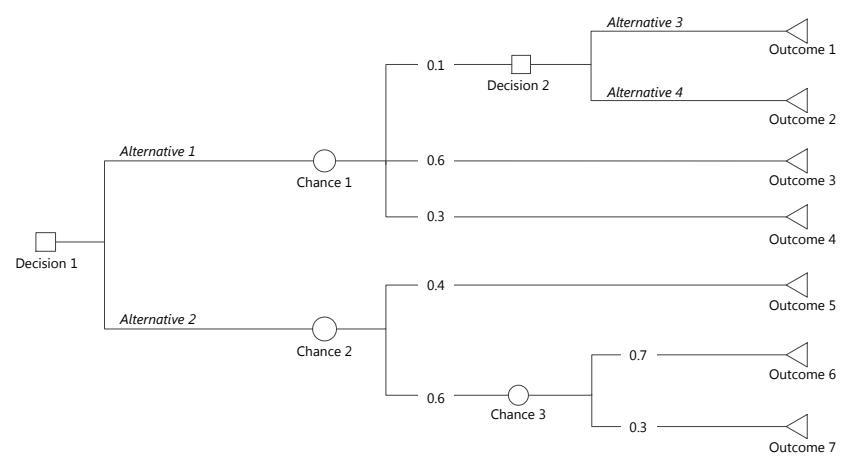

Fig. 3. Example decision tree

Influence diagrams were first suggested during the 1970 s $[32$, p. 5] [33, p. 144]. They were developed to enable formal reasoning on probabilistic relations [34, p. 127], while also being able to support communication with non- 


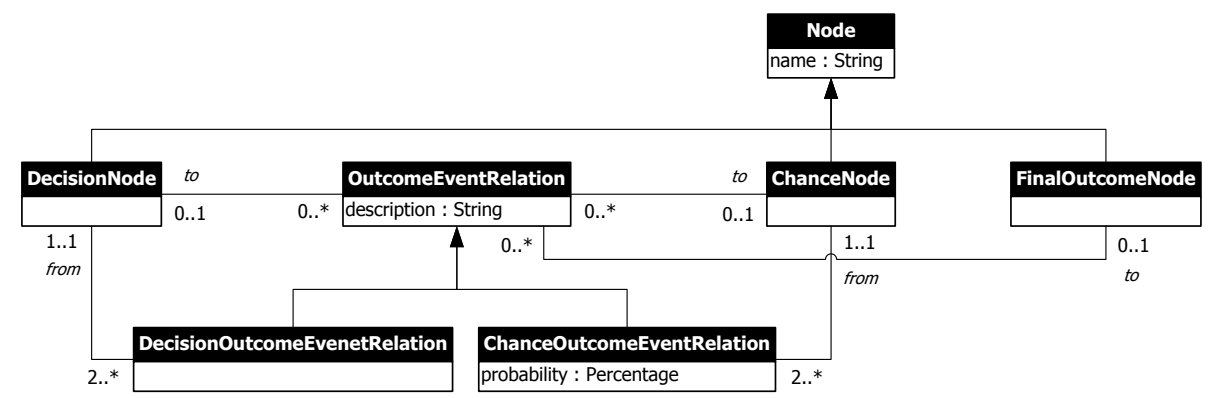

Fig. 4. Reconstructed meta model of decision trees

mathematically trained people [34, p. 127]. Influence diagrams are significantly more compact than decision trees [33, p. 144], and they especially support probabilistic operations which are hard to do using decision trees [34, pp. 129-130]. Influence diagrams consist of two basic kind of nodes: Decision nodes and chance nodes [34, p. 133]. Decision nodes describe mathematical variables whose value can be chosen. Chance nodes describe stochastic variables [34, p. 133] whose value cannot be controlled. There are two special cases of chance nodes. A deterministic node is a chance node whose conditional probability has a value of one $[32$, p. 5]. A value node, in turn, is a deterministic node whose value is to be optimized $[32$, p. 6]. Arrows that point to chance nodes state that the stochastic variable is conditionally dependent on the result of the preceding chance or decision node [34, p. 133]. Arrows heading into decision nodes mean that the outcome of the preceding variable is known [34, p. 133]. Importantly, the absence of a link means that there is probabilistic independence or ignorance of a given outcome [34, pp. 130-131]. An example of an influence diagram is shown in figure 5. Because influence diagram describe probabilistic dependencies, they can be used for advanced calculations (see [34]). In general, it is evident that influence diagrams are more abstract than decision trees and matrices. They abstract from concrete values of variables (e.g., alternatives), essentially offering a way of specifying relations among choice and random variables. Figure 6 shows a reconstructed meta model for influence diagrams.

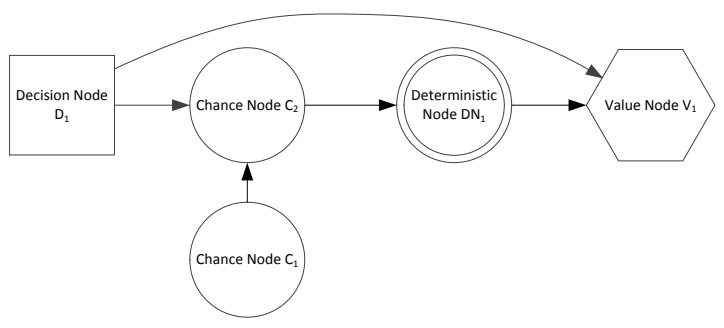

Fig. 5. Example influence diagram 


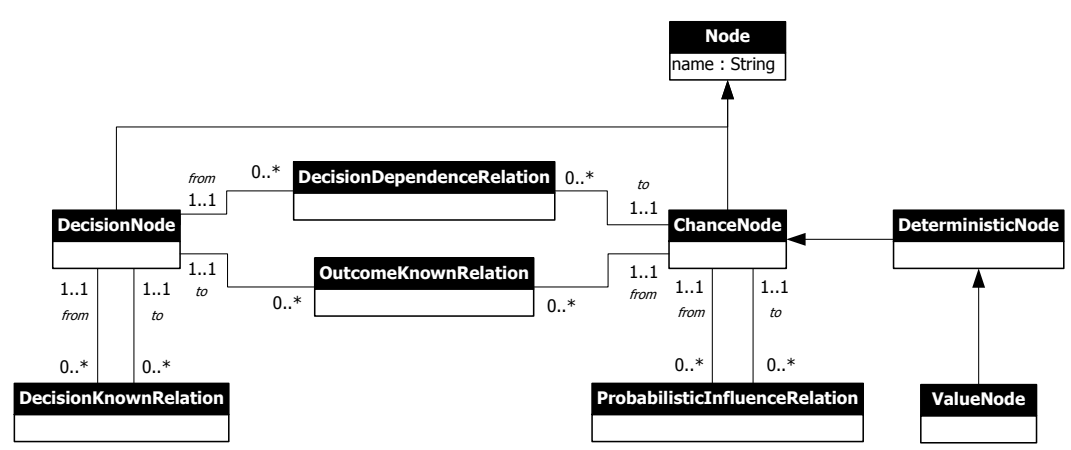

Fig. 6. Reconstructed meta model of influence diagrams

\subsection{Concepts in Problem Structuring Approaches}

As the traditional instruments of decision analysis have received a substantial share of criticism [25] [12] [26], other roads to aid decision making have been assessed. One stream of research placed emphasis on developing means to support the elicitation of different problem interpretations, communication among all stakeholders, and collaborative model-building [25, pp. 9-17] [12, pp. 106-110]. Methods from this field have become known as 'problem structuring methods' [25, pp. 9-10], and they are sometimes opposed to 'hard' approaches as 'soft' ones $[12$, p. 107]. Two common examples are visited below.

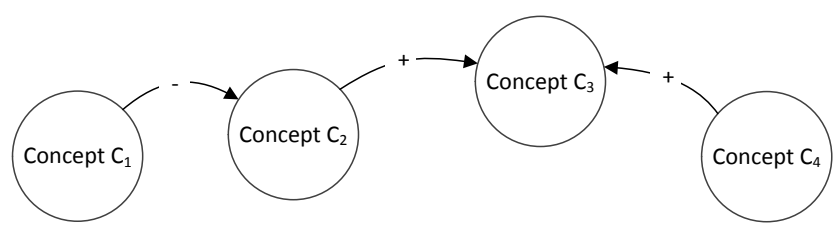

Fig. 7. Example cognitive map

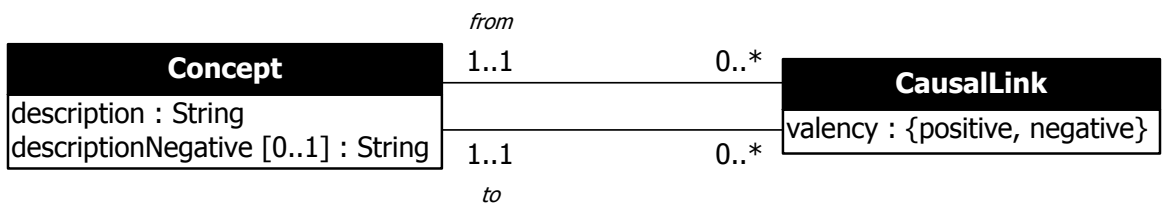

Fig. 8. Reconstructed meta model of cognitive maps 
Cognitive mapping is a modeling approach that has been used in quite distinct fields of research, including cognitive psychology, practical business consulting (see, e.g., [3] [12]), and political studies (see, e.g., [18]). It is also a part of more comprehensive methods (see [12, pp. 138-168]). Conceptually, cognitive mapping is a minimalist approach. It consists of a single concept, whose name is in fact 'concept' [12, pp. 142-143], and one relation, usually called arrow or arc [12, pp. 142-143]. A 'concept' in cognitive mapping has been defined as "an idea, issue or concern that has been expressed by someone or agreed by a group" [12, p. 142]. The arrow, in turn, expresses a causal relationship [3, p. 41] [18, pp. 5-8]. An increase (or decrease) in what is expressed by a given concept is supposed to also increase (or decrease) what is expressed by a successor. Figure 7 shows an example. It is apparent that the construct of a 'concept' is a loaded one. It is excessively broad and abstract. In consequence, quite varied aspects can be indicated by means of a cognitive map. But it is interesting to note how different the general idea is from decision analysis. Rather that selecting an "optimal" alternative, the aim is to "articulate thinking, and see it reflexively in a model [as a] representation of a part of the world as a particular person sees it" [3, pp. 43-44]. Figure 8 shows a reconstructed meta model.

Soft Systems Methodology was initially conceived in the 1970s, and it has since then been advanced and modified over time [16, p. 12]. The motivation for its inception was the observation that some assumptions of methods from the fields of decision analysis and operations research proved inadequate for the treatment of real-world problems [17, pp. 195-200]. For example, a point of criticism was directed at the assumption that all possible alternatives and consistent goal systems are already given $[25$, p. 6] (cf. section 4.1). One of the seminal ideas of SSM is to apply a "soft" notion of systems to look at problematic situations [16, p. 14]. For that purpose, the concept of a 'human activity systems' was set forth $[16$, p. 14]. It is seen as a set "of linked activities which together could exhibit the emergent property of purposefulness" [16, p. 14]. To guide the use of this idea, SSM offers a comprehensive framework for thinking. Its different components cannot be discussed fully here (for overviews, see [16] [17]). What is central from a modeling perspective is that SSM advises to create conceptual models of problem situations as interlinked human activity systems [17, pp. 218-225]. An example is provided in figure 9 . What are the concepts of such models? Besides the concept of an 'activity system' (see above), the second main concept is a 'purposeful activity' that describes "a transformation process, one in which some entity [...] is transformed into a different state" [17, p. 219]. Further, activities may be linked to "indicate the dependency of one activity upon another" $[17$, p. 223]. A reconstruction of these concepts is shown in figure 10 . When it comes to using conceptual models of activity systems, the models, again, are not intended to imply "optimal" solutions. Instead, it is emphasized that each model will be based on a particular worldview so that it will present a subjective way of seeing the problem [17, pp. 204-205]. The essential aim, then, is to support "discussion [...] to find changes which are both arguably desirable and also culturally feasible in this particular situation" [17, p. 207]. 
Taken together, it is apparent that SSM, like cognitive mapping, uses a rather small set of concepts to incite reflections on problem situations. The modeling concepts are generic in nature and do not even include include concepts to describe possible solutions. The potential usefulness of the approach for tackling ambiguous problems in social settings has been indicated in a variety of practical use cases (cf. [16, pp. 11-13]). At the same time, from a modeling perspective, SSM has been criticized for being simplistic and minimalist [35, pp. 127-128]. This, however, may be a starting point for assessing integration potentials with enterprise modeling, to which the discussion will now turn.

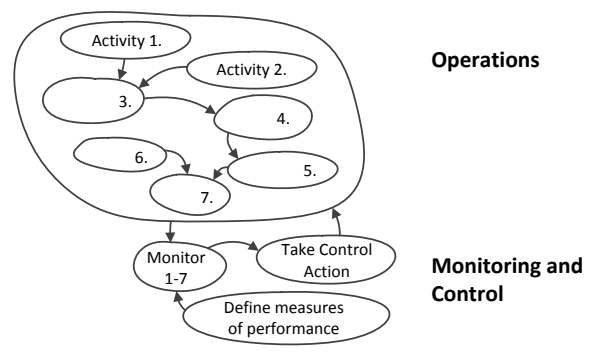

Fig. 9. Example activity system model (based on [17, p. 204])

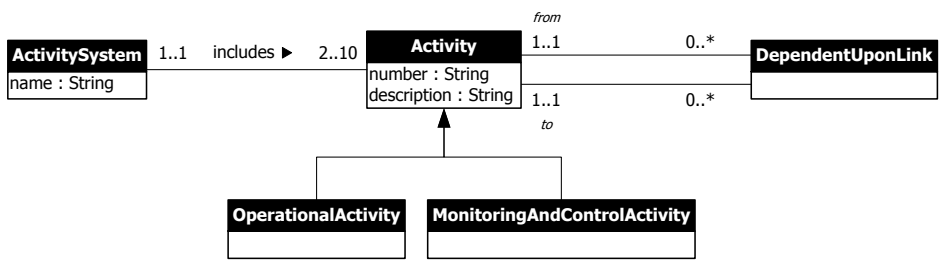

Fig. 10. Reconstructed meta model of activity systems as part of SSM

\section{Implications and Prospects}

The analysis indicated that a considerable spectrum of concepts can be used to make sense of problem situations. In this section, implications and integration points for the field of EM are discussed (section 5.1) and illustrated (section 5.2).

\subsection{General Discussion}

Key aims and integration points. As has become apparent, the aim of decision support can be taken in different ways. At one end of the spectrum, it can mean 
reasoning on concrete courses of action (see subsection 4.1). At the other end of the spectrum, it can mean aiding discussions on how to structure, understand, and reconcile different views of a problem (see subsection 4.2). While the former aim may be primarily associated with situations that are more routinized or pre-analyzed, the latter may be preferable for dealing with more ambiguous, complex, or novel problems (see [25, pp. 9-12]). In principle, either sort of decision support could be usefully advanced by incorporating decision concepts in enterprise models. Generally speaking, such an integration could mean to prepare models of certain kinds of decision situations, to link them to the location of an enterprise model where they may typically occur, and to critically reassess and adjust them periodically. This could contribute to improving organizational decision making practices over time. Furthermore, the use and interpretation of the decision models may profit from the domain-specific knowledge in the enterprise model (see below). Organizational areas where rule-driven or preanalyzable decision situations may naturally occur include operational business processes (see, e.g., [30, pp. 19-20]) and other operational areas of responsibility. Consequentially, models based on choice-centric concepts (e.g., those of decision matrices or influence diagrams) could be attached to branching points, activities, or task concepts in an EM modeling language. The practical use of these models might consist in analyzing recurring choice situations during operational processes. Steps in this direction have already been made, although the focus so far has been placed on prescribing rather than aiding decision making (cf. [10, pp. 19-21]). With respect to decision processes that deal with more complex and ambiguous problems, EM languages could be augmented with broader concepts such as those identified in section 4.2. Model use would then shift to aiding discussions and explorations into the unclear situations in question. However, in contrast to operational decisions, it is not obvious how these models could be integrated with EM methods, as there are few concepts to describe non-routine decision processes (but see section 3 and [8] for a suggestion in this direction). In other words, adequate abstractions are needed.

Adequate abstractions. To develop time-stable models of non-routine decision situations or decision processes at level $M_{1}$, it is necessary to develop abstractions of similar past, present, and future occurrences of these situations or processes (see also [8, pp. 184, 187]). However, non-routine decision situations are precisely characterized by novelty or ambiguity. This is a challenge for future research.

Domain-specificity. The analysis has shown that the concepts which are used in traditional decision tools are essentially generic in nature (e.g., 'alternative', 'concept', and 'activity'). They can be used to describe choices in almost any domain. Using highly abstract and generic concepts to understand decision making, in fact, has a long tradition in decision research, as is demonstrated by the widely shared choice view (see [21, pp. 568-572]). Contrary to this position, however, results in psychological research indicate that people who deal with decision situations draw on, and require, domain-specific knowledge, expertise, and concepts [21, pp. 581-601]. Therefore, an immediate value of enterprise modeling can be seen in the fact that it offers a rich source of domain specific knowledge, 
from the instance level $M_{0}$ (i.e., knowledge about particular occurrences) to the (meta) level $M_{2}$ or higher (i.e., conceptual knowledge). A first step of utilizing this prospect may consist in integrating decision models in enterprise models at level $M_{1}$ (as noted above). But beyond such a straightforward integration, it would also seem promising to investigate how the domain-specific knowledge captured in an enterprise model and its linguistic structure at level $M_{2}$ or above could be exploited to facilitate more sophisticated deliberation processes.

Ways of seeing. Finally, the analysis revealed that different decision modeling approaches present fundamentally different ways of seeing a problem situation (cf. [12, p. 106]). The approaches from the field of decision analysis essentially take an instrumental means-end perspective (cf. [25, pp. 6-7] [27, p. 321]). A choice must be made among given means (alternatives) such as to achieve given ends (goals) [25, p. 7]. Cognitive maps, in turn, consider problem situations solely in terms of causal relations. This permits to describe aspects other than those captured by the choice framework - but only in causal terms, which itself excludes other aspects (e.g., teleological ones). The perspective of SSM, in turn, gives weight to the purposefulness of human activities in problem situations [17, p. 192]. In contrast to the first perspective, this does not presuppose a solution space of possible means. As a general conclusion, it may be stated that any set of decision modeling concepts can bring about a specific way of seeing problem situations that highlights some aspects and forgoes others. "The language we used is not neutral and can even become a trap, confusing [...] the way [of] talking about unsatisfactory situations with reality itself" [27, p. 339]. That seems important, too, when thinking about modeling languages.

\subsection{Illustration of an Example Integration}

It has been indicated that enterprise modeling could possibly provide a helpful basis for decision support. Finally, in order to indicate how an augmentation of existing EM methods with classical concepts could look like, a simple scenario is illustrated in figure 11. The figure displays four partial diagrams of the enterprise model of a production company. The model is created using various MEMO languages [5] [29] [30]. It consists of (1) a partial goal model, (2) a model of the organizational structure, (3) a model of organizational decision processes, (4) and a model of a business process. The preliminary concept and notation of a decision process type (the blue hexagons) are adopted from [8]. Two decision process types can be found: One is concerned with routine decisions in the business process (automatic vs. manual production), whereas the other is dealing with a more complex problem (reconsidering and possibly restructuring the entire production process). The key point is that these decision process types are linked to partial classical decision models. In line with the nature of the decision processes, the more routinized one is detailed by means of a decision matrix. For the less structured and predetermined one, an excerpt of a cognitive map is attached, which could be used to reflect on the assumed causal relations.

What are the prospects of such an integration? First, and most importantly, embedding the classical models with enterprise models enriches them with in- 

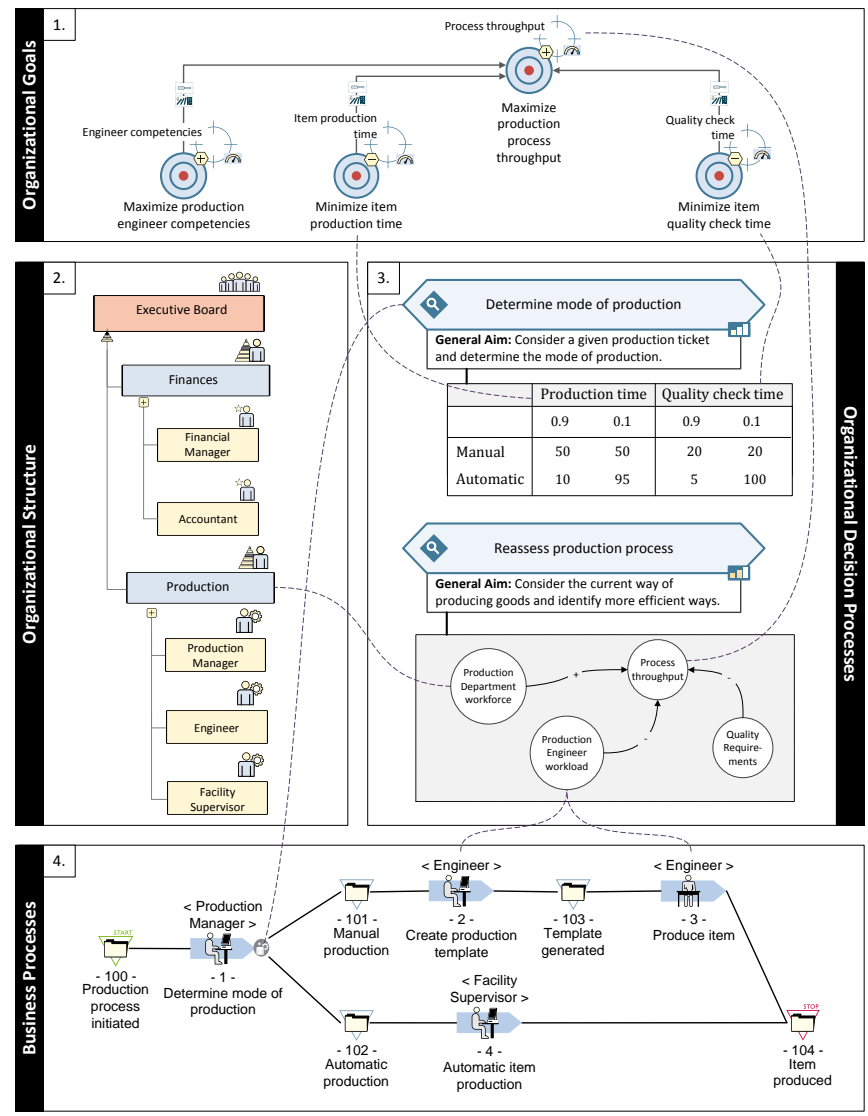

Fig. 11. Illustration of an example integration

formation on the relevant context. This is indicated by the violet dotted lines. For example, the goals which are considered in the decision matrix in fact correspond to goals specified in the organizational goal system (see partial diagram 1). As another example, the different concepts in the cognitive map are linked to different elements of the enterprise model, such as units of the organizational structure (partial diagram 3), or activities in the business process (partial diagram 4). This can be assumed to foster interpretation of the decision situation, while also offering connection points for further analyses in the enterprise model. Furthermore, it would be possible to augment the links with additional attributes that could be used to make explicit the underlying assumptions, or to note estimations regarding their validity. Finally, in case such integrated models would be maintained over time, they could be used as part of continuous management processes to reassess, and perhaps improve decision making practices. 


\section{Conclusions and Outlook}

Decision making is at the heart of human action. An analysis of classical modelbased approaches has brought to the fore quite distinct conceptual ways of dissecting decision situations. Apparent linking points and complementary benefits, as well as general prospects and challenges of enriching the field of enterprise modeling with decision concepts have been discussed. Various paths for future research can be suggested. First, it would generally seem promising to advance the use of EM for purposes of decision support, both in the long term and for specific situations. In particular, support for decision processes dealing with complex, ambiguous, or novel problems can be greatly enhanced. Second, further attention could be directed at the potential role of enterprise models as stocks of domain-specific (conceptual) knowledge in decision processes. Third, to realize decision models that are useful in the long term, it seems necessary to advance conceptions of type abstractions of decision situations or processes. Finally, the analysis has also indicated that awareness may be raised on the possible limits and biases of the language used to speak about decisions.

\section{References}

1. March, J.G.: Understanding How Decisions Happen in Organizations. In March, J.G., ed.: The Pursuit of Organizational Intelligence. Blackwell, Malden (1999) $13-38$

2. Keeney, R.L.: Decision analysis: An overview. Op. Research 30(5) (1982) 803-838

3. Eden, C., Jones, S., Sims, D.: Messing About in Problems: An Informal Structured Approach to their Identification and Management. Pergamon Press, Oxford (1983)

4. Yates, J.F.: Decision Management: How to Assure Better Decisions in Your Company. Jossey-Bass, San Francisco (2003)

5. Frank, U.: Multi-perspective enterprise modeling: foundational concepts, prospects and future research challenges. Software \& Systems Modeling 13(3) (2014) 941-962

6. Sandkuhl, K., Stirna, J., Persson, A., Wißotzki, M.: Enterprise Modeling: Tackling Business Challenges with the 4EM Method. Springer, Berlin, Heidelberg (2014)

7. The Open Group: ArchiMate 2.0 Specification: Open Group Standard. The Open Group Series. Van Haren, Zaltbommel (2012)

8. Bock, A.C.: Beyond Narrow Decision Models: Toward Integrative Models of Organizational Decision Processes. In: Proceedings of the 17th IEEE Conference on Business Informatics (CBI 2015), IEEE Computer Society (2015) 181-190

9. Plataniotis, G., Kinderen, S.d., Proper, H.A.: Capturing design rationales in enterprise architecture: A case study. In Frank, U., Loucopoulos, P., Pastor, Ó., Petrounias, I., eds.: The Practice of Enterprise Modeling. Springer, Heidelberg and New York and Dordrecht and London (2014) 133-147

10. Object Management Group: Decision Model and Notation: Beta 1 (2014)

11. Davenport, T.H.: Make Better Decisions. HBR 87(11) (2009) 117-123

12. Pidd, M.: Tools for Thinking: Modelling in Management Science. 2 edn. Wiley, Chichester and Hoboken (2003)

13. Triantaphyllou, E.: Multi-Criteria Decision Making Methods: A Comparative Study. Kluwer, Dordrecht, Boston (2000) 
14. Raiffa, H.: Decision Analysis: Introductory Lectures on Choices under Uncertainty. Addison-Wesley, Reading (1970)

15. Bamberg, G., Coenenberg, A.G., Krapp, M.: Betriebswirtschaftliche Entscheidungslehre. 14 edn. Vahlen, München (2008)

16. Checkland, P.: Soft Systems Methodology: A Thirty Year Retrospective. Systems Research and Behavioral Science 17(1) (2000) S11-S58

17. Checkland, P., Poulter, J.: Soft systems methodology. In Reynolds, M., Holwell, S., eds.: Systems Approaches to Managing Change. Springer, London (2010) 191-242

18. Axelrod, R.: The cognitive mapping approach to decision making. In Axelrod, R., ed.: Structure of Decision. Princeton University Press, Princeton (1976) 3-17

19. Elbing, A.O.: Behavioral Decisions in Organizations. Scott, Foresman and Company, Glenview (1970)

20. Hastie, R.: Problems for judgment and decision making. Annual Review of Psychology 52 (2001) 653-683

21. Goldstein, W.M., Weber, E.U.: Content and discontent: Indications and implications of domain specificity in preferential decision making. In Goldstein, W.M., Hogarth, R.M., eds.: Research on Judgment and Decision Making. Cambridge University Press, Cambridge and New York (1997) 566-617

22. Simon, H.A.: Administrative Behavior: A Study of Decision-Making Processes in Administrative Organization. 3 edn. Free Press, New York (1976)

23. Simon, H.A.: The New Science of Management Decision. Harper, New York (1960)

24. Mintzberg, H., Raisinghani, D., Théorêt, A.: The structure of unstructured decision processes. Administrative Science Quarterly 21(2) (1976) 246-275

25. Rosenhead, J., Mingers, J.: A new paradigm of analysis. In Rosenhead, J., Mingers, J., eds.: Rational Analysis for a Problematic World Revisited. Wiley, Chichester and New York (2001) 1-19

26. Bretzke, W.R.: Der Problembezug von Entscheidungsmodellen. Mohr, Tübingen (1980)

27. Landry, M.: A Note on the Concept of 'Problem'. Organization Studies 16(2) (1995) 315-343

28. Bock, A.C., Kaczmarek, M., Overbeek, S., Heß, M.: A comparative analysis of selected enterprise modeling approaches. In Frank, U., Loucopoulos, P., Pastor, Ó., Petrounias, I., eds.: The Practice of Enterprise Modeling. Springer, Heidelberg and New York and Dordrecht and London (2014) 148-163

29. Overbeek, S., Frank, U., Köhling, C.: A language for multi-perspective goal modelling: Challenges, requirements and solutions. Computer Standards \& Interfaces 38(February) (2015) 1-16

30. Frank, U.: MEMO Organisation Modelling Language (2) - Focus on Business Processes. ICB Research Report 49, University of Duisburg-Essen, Essen (2011)

31. Frank, U.: The MEMO Meta Modelling Language (MML) and Language Architecture. ICB Research Report 43, University of Duisburg-Essen, Essen (2011)

32. Howard, R.A.: From influence to relevance to knowledge. In Oliver, R.M., Smith, J.Q., eds.: Influence Diagrams, Belief Nets and Decision Analysis. Wiley, Chichester (1990) 3-23

33. Howard, R.A., Matheson, J.E.: Influence diagram retrospective. Decision Analysis 2(3) (2005) 144-147

34. Howard, R.A., Matheson, J.E.: Influence diagrams. Decision Analysis 2(3) (2005) 127-143

35. Hirschheim, R., Klein, H.K., Lyytinen, K.: Information Systems Development and Data Modeling: Conceptual and Philosophical Foundations. Cambridge University Press, Cambridge (1995) 